\title{
Preoperative Predictors of Postoperative Cognitive Dysfunction after Y-graft Replacement for Abdominal Aortic Aneurysm
}

M Hayashi, MD, T Baba, MD. K Maekawa, MD, Y Tokunaga, MD, and N Miyagawa, MD Department of Anesthesiology, Kumamoto Chuo Hospital, Kumamoto, Japan

\section{Background}

Postoperative cognitive dysfunction (POCD) is believed to result from the stress associated with operations.

Although Y-graft replacement is a highly invasive procedure and should lead to an elevated rate of subsequent POCD, there are only a few reports of preoperative predictors of POCD after Y-graft replacement.

\section{Objective}

To identify the preoperative predictors of POCD after Y-graft replacement.

\section{Materials \& Methods}

Study population: elective 65 patients (72.3 \pm 7.0 ys) underwent Y-graft replacement for Abdominal aortic aneurysm (Jul.2010 - Apr.2015)

Anaesthesia:

- Either general anesthesia or combined general and epidural anesthesia

Neurological evaluation: preop and 7 days postop

Hasegawa Dementia Scale : HDS, modification of MMSE, full score 30

Kana pick-up test

letter cancellation test, full score 60

Digit Symbol test

Digit Span test

-POCD was defined as a decrease of at least $20 \%$ from baseline

for an individual's performance in more than one test.
Statistical analysis:

- Unpaired t-test, and chi-square test or Fisher's exact test

$\mathrm{P}<0.05$ was considered statistically significant.

- Stepwise logistic regression analysis

The level of significance for the univariate screening regressions was set at $\mathrm{P}<0.2$

The ROC curves were depicted and the cut-offs were determined by the shortest distance from the top left corner of the curve.

- Comparison of POCD group and non POCD group characteristics and perioperative factors.

- Evaluate the probability of POCD for each combination of risk factors.

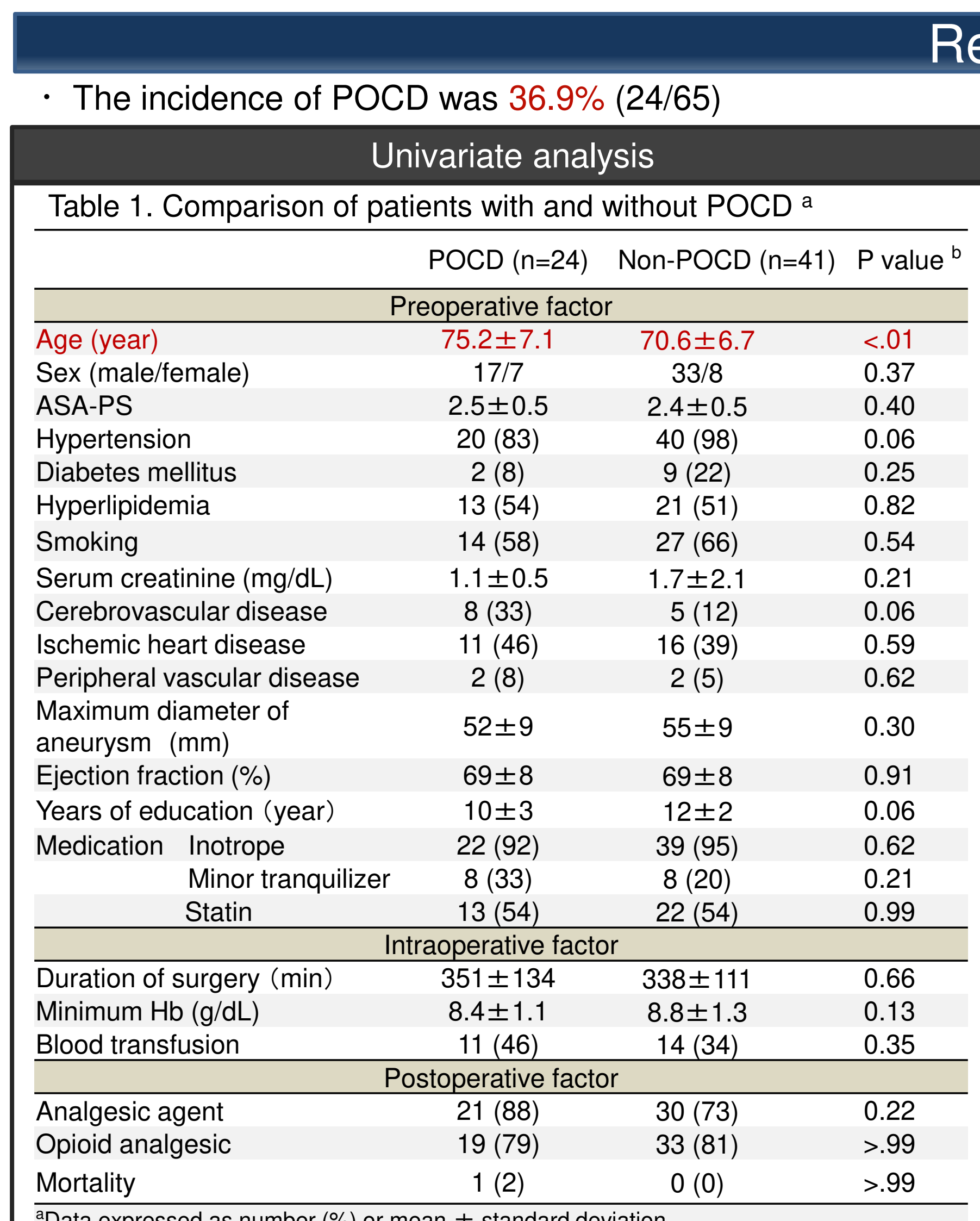

${ }^{a}$ Data expressed as number (\%) or mean \pm standard deviation.

${ }^{\mathrm{b}} \mathrm{P}$ values refer to comparisons between groups.

Figure1. Preoperative cognitive tests

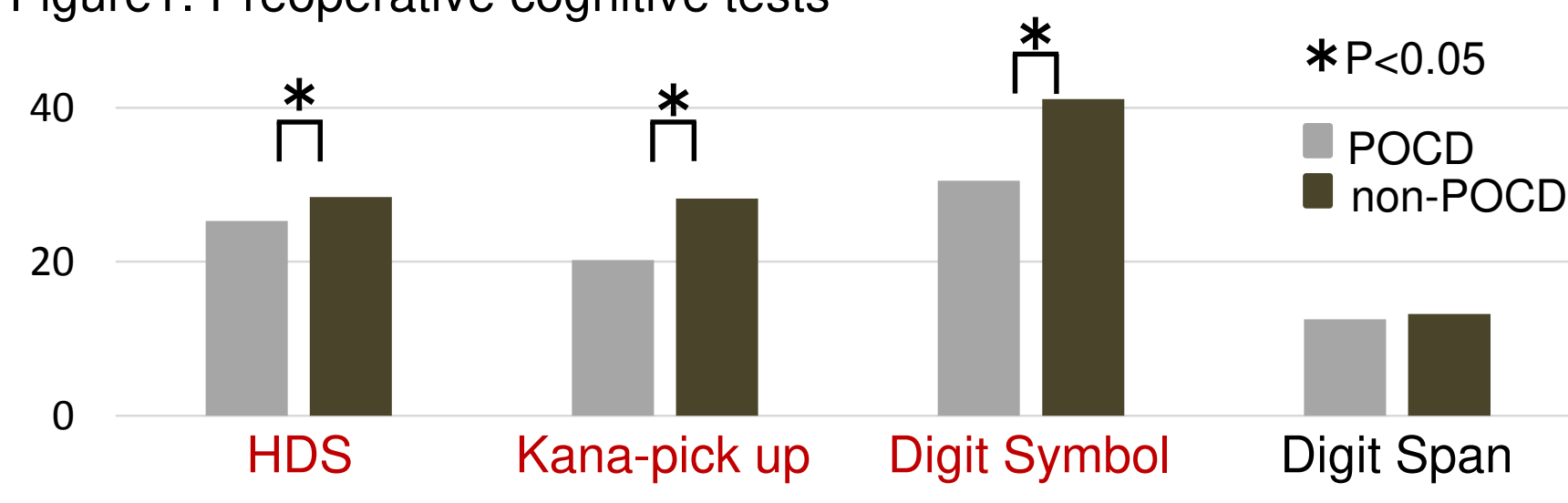

POCD group was significantly older,

had lower neuropsychological scores.

(HDS, Kana pick-up test, and Digit symbol test)
Table2. Cut-offs values for POCD risk

\begin{tabular}{|c|c|c|c|c|}
\hline & Cut-offs value & sensitivity $s$ & specificity & AUC \\
\hline Age (year) & 74 & 0.667 & 0.737 & 0.702 \\
\hline Minimum $\mathrm{Hb}(\mathrm{g} / \mathrm{dL})$ & 8.6 & 0.583 & 0.526 & 0.555 \\
\hline Schooling history (year) & 9 & 0.583 & 0.711 & 0.634 \\
\hline HDS & 28 & 0.833 & 0.632 & 0.796 \\
\hline Kana pick-up test & 26 & 0.708 & 0.500 & 0.655 \\
\hline Digit Symbol test & 34 & 0.708 & 0.737 & 0.751 \\
\hline $\begin{array}{l}\text { AUC: area under the curve, HDS: } \\
\text { Table 3. Multivariate analy }\end{array}$ & $\begin{array}{l}\text { Hasegawa Dement } \\
\text { lysis }\end{array}$ & a Scale & & \\
\hline & OR & $95 \% \mathrm{Cl}$ & $P$ value & \\
\hline CVD & 3.04 & $0.66-14.09$ & 0.15 & \\
\hline Age $\geq 74$ ys & 4.47 & $1.30-15.31$ & 0.02 & \\
\hline HDS $\leq 28$ & 7.76 & $2.05-29.33$ & $<.01$ & \\
\hline $\begin{array}{l}\text { CVD: Cerebrovascular disease } \\
\text { HDS: Hasegawa Dementia Scale }\end{array}$ & \multicolumn{4}{|c|}{$\begin{array}{l}\text { OR: odds ratio } \\
\text { Cl: confidence interval }\end{array}$} \\
\hline
\end{tabular}

Figure2. The probability of POCD for each combination of risk factors

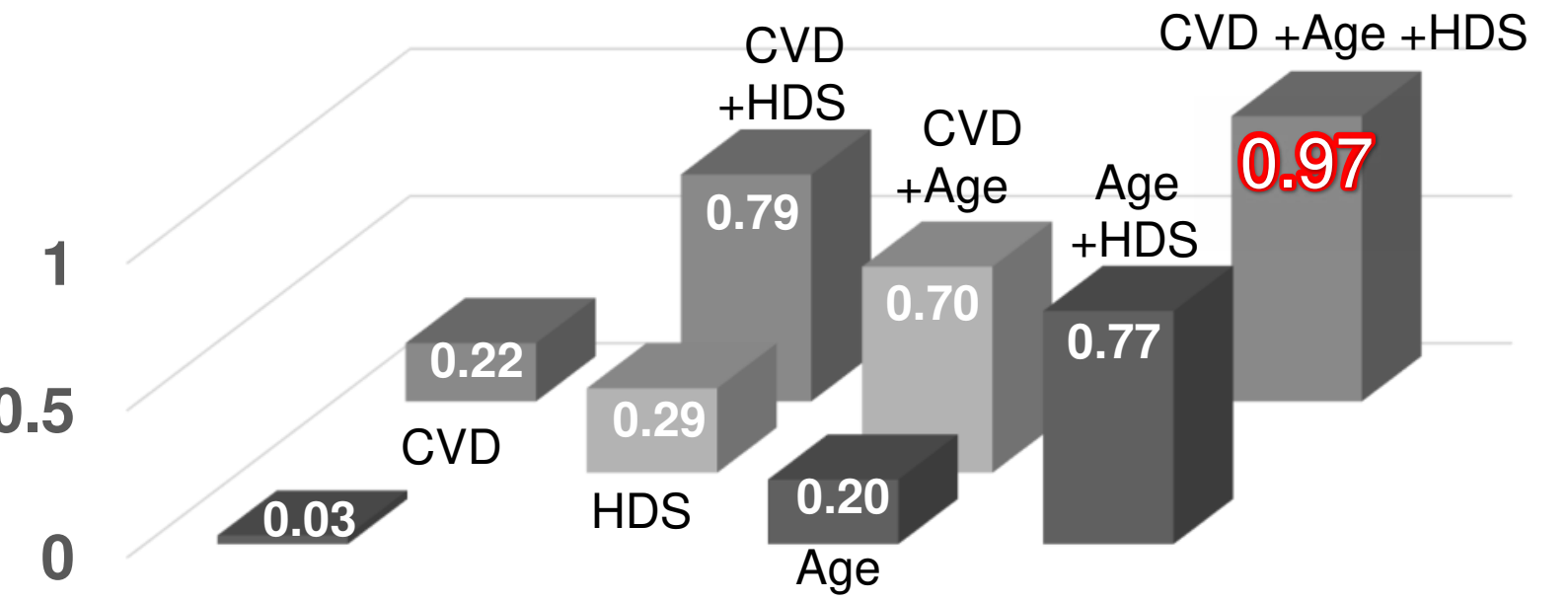

CVD: Cerebrovascular disease, Age: Age $\geq 74$ ys, HDS: Hasegawa Dementia Scale score $\leq 28$

- The probability of POCD in AAA patients who underwent Y-graft replacement with CVD, Age $\geq 74$ ys, and HDS score $\leq 28$ was 0.97

\section{Conclusion}

- The incidence of POCD after Y-graft replacement was 36.9\% (24 / 65).

- Cerebrovascular disease, age $\geq 74$ ys, HDS score $\leq 28$ were independent preoperative predictors of POCD. These factors represent decreased cognitive reserve, and would be associated with POCD

- HDS or MMSE may be useful to predict POCD before surgery in elderly patient with history of CVD. 